\title{
Uma Abordagem Para Detecção de Nódulos Pulmonares Baseada em Aprendizado Profundo
}

\author{
Émerson Silva $^{1}$, Luís França ${ }^{1}$, Marcelo Oliveira ${ }^{2}$, Fabiane Queiroz $^{3}$, Rodolfo Cavalcante ${ }^{1}$ \\ ${ }^{1}$ Núcleo de Ciências Exatas - Universidade Federal do Alagoas \\ CEP 57309-005 Arapiraca - AL - Brazil \\ ${ }^{2}$ Instituto de Computação - Universidade Federal de Alagoas \\ CEP 57072-900 - Maceió - AL.
}

${ }^{3}$ Campus de Enhenharias e Ciências Agrárias - Universidade Federal de Alagoas CEP 57100-000 - Rio Largo - AL.

\{emerson.silva, luis.franca, rodolfo.cavalcante\}@arapiraca.ufal.br,

oliveiramceic.ufal.br,fabiane.queirozececa.ufal.br

\begin{abstract}
Lung cancer is the kind of cancer which causes the higher number of deaths in the world. This justifies the need of a fast identification. The diagnosis of lung cancer is performed by specialists by means of computed tomography (CT) scans of the patients chest. This diagnosis is made in two important stages: (i) the detection of existing nodules and (ii) the classification of these nodules into malignant or benign, if they exist. In this work we investigated how to detect lung nodules in CT 2D by using convolutional neural networks (CNN) and principal component analyis (PCA). The main contribution of this work is a detection method applied to $2 D C T$ scans which presents an accuracy similar to methods applied to 3D imagens, which are consequently more computationaly expensive. Results presented $86 \%$ of sensibility in identifying lung nodules.
\end{abstract}

Resumo. O cancêr de pulmão é o tipo de câncer que mais causa mortes no mundo, o que justifica a necessidade de uma rápida identificação. Os diagnósticos do câncer de pulmão podem ser realizado por especialistas através da análise de imagens de tomografia computadorizada (TC) do tórax do paciente. Esse diagnóstico envolve duas tarefas importantes: (i) a detecção de nódulos existentes e (ii) a classificação destes nódulos em maligno ou benigno, caso existam. Neste trabalho investigamos como detectar nódulos pulmonares em imagens de TC $2 D$ com o uso de redes neurais convolucionais (CNN) e análise de componentes principais (PCA). A principal contribuição desse trabalho é um método de detecção aplicado a imagens de TC $2 D$ que apresenta acurácia próxima a métodos aplicados a imagens $3 D$ e, consequentemente computacionalmente mais caros. Os resultados apresentaram $86 \%$ de sensibilidade na identificação de nódulos pulmonares. 


\section{Introdução}

Entende-se por câncer um conjunto de doenças que se caracterizam pelo crescimento anormal e incontrolado de células que invadem tecidos e órgãos vizinhos [Cesár Uehara 1998]. O câncer de pulmão é considerado o tipo de câncer que mais causa mortes no mundo, com incidência de aproximadamente 1,2 milhões de casos por ano e com taxa de mortalidade de 1 milhão de pacientes por ano [Cesár Uehara 2008]. Sua principal manifestação ocorre devido ao aparecimento de lesões no tecido pulmonar. Dada a sua alta taxa de mortalidade, faz-se necessário que esse tipo de câncer seja identificado e diagnosticado o mais rápido o possível. Esse diagnóstico é tipicamente realizado por especialistas através da análise de vídeos/imagens de Tomografia Computadorizada (TC) do tórax do paciente. A maior dificuldade nesta tarefa está em distinguir nódulos verdadeiros de outras estruturas pulmonares ou de outros órgãos e tecidos. Aliado a isso, aspectos humanos podem interferir da identificação dos nódulos por parte dos especialistas, como cansaço, pressa, inexperiência, dentre outros fatores psicológicos [Bogot et al. 2005].

Neste contexto, técnicas de visão computacional podem ser capazes de auxiliar na identificação de nódulos em imagens de TC da região torácica. Atualmente, algumas técnicas de aprendizado de máquina profundo (Deep Learning) têm sido aplicadas com relativo sucesso em tarefas de análise de imagens médicas e auxílio ao diagnóstico por imagem [Shen et al. 2017, Ker et al. 2017, Rakhlin et al. 2018]. Mais especificamente, Redes Neurais Convolucionais (Convolutional Neural Networks - CNNs) têm sido usadas para assistir à identificação de nódulos pulmonares em imagens tomográficas de maneira eficiente [Xie et al. 2019, Eun et al. 2018, Jiang et al. 2018]. Tais técnicas são capazes de avaliar vários aspectos da imagem como: textura, densidade, tamanho das estruturas, e etc. Também é possível, através da correlação das informações das imagens analisadas, realizar o reconhecimento de padrões, o que permite obter uma maior precisão no resultado final do diagnóstico. A automatização da identificação de nódulos evita a interferência de fatores humanos e pode servir como segunda opinião em um diagnóstico médico.

Neste artigo propomos uma solução eficiente para detecção de nódulos em imagens bidimensionais de TC. O método aqui proposto é baseado na segmentação automática da região do parênquima pulmonar, seguida da detecção de possíveis nódulos internos a esta região. A segmentação do parênquima é realizada com o treinamento de uma rede neural do tipo U-net [Ronneberger et al. 2015] para realizar a segmentação. A identificação dos nódulos é então realizada com o uso de uma rede neural convolucional. A principal contribuição deste trabalho reside na etapa de detecção do nódulo, definida pelo uso de uma CNN agregada à redução de dimensionalidade de dados utilizando o método de Análise de Componentes Principais (Principal Component Analysis - PCA) [Dunteman 1989]. Desta forma, com o objetivo de diminuir a redundância das características extraídas pela CNN, foi aplicado o PCA na detecção do possíveis nódulos.

O restante deste artigo está organizado como segue. $\mathrm{Na}$ Seção 2, apresentamos os trabalhos relacionados, onde serão apresentados alguns estudos que utilizam abordagens de Deep Learning para a análise de imagens de tomografia computadorizada para detectar o câncer de pulmão. Na Seção 3, apresentamos o método proposto para detecção de nódulos pulmonares em imagens de TC. Na Seção 4.4, são descritos os experimentos realizados e resultados obtidos com o método proposto, onde são também detalhadas 
informações sobre bases de dados utilizadas, bem como sobre as métricas e formas de validação dos experimentos e dos resultados alcançados. As conclusões e os trabalhos futuros são apresentados na Seção 5.

\section{Trabalhos Relacionados}

Métodos de aprendizado de máquina têm sido largamente utilizados na identificação de nódulos pulmonares por apresentarem resultados promissores [Chen et al. 2019]. Os trabalhos aqui abordados propuseram métodos que utilizam CNNs para realizar a extração de informações de tomografias computadorizadas da região do tórax. Tais trabalhos são definidos em contextos de imagens 2D [Xie et al. 2019, Eun et al. 2018, Lakshmanaprabu S.K. 2018] ou 3D [Golan et al. 2016, Gu et al. 2018, Huang 2017, Ding 2019, Winkels and Cohen 2019]. Nesta seção, descrevemos alguns trabalhos relacionados considerando diferentes aspectos, como métodos utilizadas, resultados alcançados e suas limitações.

Dentre trabalhos recentes no contexto de imagens 2D, em [Xie et al. 2019] foi utilizado o modelo Faster $R$-CNN [Ren et al. 2015] na base de dados de imagens LUNA16 [Setio et al. 2017], com o objetivo de identificar possíveis regiões contendo nódulos nas imagens, seguida da aplicação de uma camada CNN 2D para a redução de falsos positivos. Nesta camada, um classificador é responsável por distinguir nódulos verdadeiros de candidatos. Com essa abordagem foi possível alcançar $86,42 \%$ de acurácia na detecção e uma taxa de $74,4 \%$ na proporção de $1 / 8$ para a redução de falsos positivos.

[Eun et al. 2018] propuseram um método que realiza extração dos possíveis candidatos a nódulos e uma classificação posterior de fragmentos de imagem (patches) nas classes nódulo e não nódulo. Neste trabalho, foi utilizado uma autoencoder e o algoritmo k-means para agrupamento dos patches e extração de candidatos a nódulos. Em seguida, os patches que possuíam grande probabilidade de pertencer a classe nódulo foram passados como entrada para uma rede CNN 2D para a redução dos falsos positivos. Os autores deste trabalho defendem que tal abordagem possui menos consumo de memória e complexidade computacional diante das estruturas com redes que trabalham com imagens 3D, alcançando o estado da arte com 0,922 de CPM (Competition Perfomance Metric score).

Em [Jiang et al. 2018] foi proposto uma abordagem que utiliza CNN 2D em um esquema em multi-escala, ao qual patches dimensionados em 4 diferentes tamanhos são enviados para a CNN com o objetivo de ampliar o poder de captação das informações contidas nas imagens. Os experimentos realizados mostraram que o método proposto alcançou $80,06 \%$ de sensibilidade com a pontuação de 4,7 falsos positivos por scan.

Recentemente, pesquisas têm se dedicado a investigar abordagens de análise de imagens 3D [Ding 2019, Winkels and Cohen 2019]. Tais trabalhos se mostram eficientes no diagnóstico de nódulos pulmonares por possuírem uma área contextual mais rica em informações. Vale ressaltar que os dados deixam de serem tratados como pixels e passam a serem analisados como voxels. [Ding 2019] apresentou um trabalho baseado no uso da Faster $R-C N N$ para a extração de candidatos a nódulos e uso de camadas convolucionais 3D para a redução de falsos positivos. Já [Winkels and Cohen 2019] apresentaram uma abordagem baseada no uso de blocos de camada de convolução, nomeado como G-CNN. Uma limitação destas abordagens 3D é o custo computacional elevado, tanto no préprocessamento das imagens, quanto no processo de treinamento das redes convolucionais 
[Golan et al. 2016].

O trabalho proposto por [Gu et al. 2018] utilizou uma abordagem multi-escala com CNN3D ao qual o método avaliou cerca de 888 scan com finas fatias com 1186 nódulos providos da base de dados LUNA16. Todos os resultados foram obtidos pela validação cruzada com o 10 -fold, alcançando assim $87,94 \%$ para 1 falso positivo por scan obtendo Competition Perfomance Metric (CPM) de 0,7967.

O trabalho proposto em [Huang 2017] traz uma abordagem ainda dentro do contexto informacional provido pela rede $\mathrm{CNN} 3 \mathrm{D}$, porém a sua diferença para os trabalhos já citados é a maneira como a análise ocorre, onde utiliza de técnicas como data augmentation para o aumento da base de dados e aplicação de regularização de dados para reduzir o sobreajuste. Para a geração de candidatos é usado um filtro baseado em modelo geométrico local e também é buscado a redução da variabilidade de estruturas estimando a orientação local. É alcançado uma boa performance, porém com algumas limitações, como: nódulos encontrados próximo ao parênquima de difícil dissociação entre estruturas do próprio pulmão com os candidatos a nódulo; regiões que sofrem opacidade devido a aparição de partes arteriais; e a redução de desempenho do sistema quando submetido ao método de validação cruzada.

Em [Chen et al. 2019] é proposto um método que combina arquiteturas 2D e $3 \mathrm{D}$, onde a primeira é utilizada para a segmentação de nódulos suspeitos a serem falsos positivos e a remoção destes, então é usado os nódulos no formato 3D para uma melhor classificação entre nódulos e não-nódulos. Este método foi aplicado com 1000 pacientes e obteve $90,1 \%$ de acurácia na detecção. Porém a integração das redes convolucionais apresentou um gargalo entre as conexões, o que causa uma certa taxa de erro que acaba por influenciar no resultado obtido.

A Tabela 1 apresenta a comparação dos trabalhos relacionados. A tabela está organizada segundo alguns critérios, a saber: (i) o uso de informações contextuais 2D(pixel), nos 4 primeiros trabalhos, e 3D(voxel) nos demais trabalhos; (i) as métricas utilizadas para avaliação de performance: Acurácia (Acc), Sensibilidade (ST), Falsos-Positivos (FP), Competition Perfomance Metric (CPM), Free-response Receiver Operating Characteristic (FROC) e o Erro Médio Quadrado (MSE); e (iii) os principais resultados obtidos.

\begin{tabular}{|c|c|c|c|c|c|}
\hline Autor & Ano & Dataset & Método & Métricas & Resultados \\
\hline [Eun et al. 2018] & 2018 & LUNA16 & Autoencoder + K-means + CNN 2D & Acurácia e Sensibilidade & Acc: $80,00 \%$, ST: $92,20 \%$ \\
\hline [Jiang et al. 2018] & 2018 & LIDC & Multi-scale CNN & Sensibilidade e False-Positive & ST: $80,06 \%$, FP: 4,7 por scan \\
\hline [Xie et al. 2019] & 2019 & LUNA16 & Faster R-CNN + CNN 2D & Acurácia e False-Positive & Acc: $86,42 \%$, FP: $1 / 8$ por scan \\
\hline [Winkels and Cohen 2019] & 2019 & NSLT + LIDC & G-CNN & FROC & FROC: 0,880 \\
\hline [Golan et al. 2016] & 2016 & LIDC + IDRI & CNN 3D & Sensibilidade False-Positive & ST: $78,9 \%$, FP: 20 por scan \\
\hline [Huang 2017] & 2017 & LIDC & CNN 3D & Sensibilidade e False-Positive & ST: $90 \%$, FP: 5 por scan \\
\hline [Gu et al. 2018] & 2018 & LIDC & CNN 3D + Data Augmentation & Sensibilidade False-Positive e CPM & ST: $87,94 \%$, FP: 1 por scan, CPM: 0,796 \\
\hline [Chen et al. 2019] & 2019 & LIDC & CNN 2D + CNN 3D & Acurácia & Acc: $90,01 \%$ \\
\hline [Ding 2019] & 2019 & LUNA16 & Faster R-CNN & FROC & FROC: 0,891 \\
\hline
\end{tabular}

Tabela 1. Tabela comparativa dos métodos fundamentados no contexto 2D(pixel) e 3D(pixel

O uso de rede CNN 2D nos permite ter resultados satisfatórios mesmo trabalhando em um contexto de menos informação, quando comparado ao contexto de imagens 3D. Um ganho importante de se trabalhar em um cenário 2D está em um menor custo computacional. Neste trabalho propomos um método capaz de identificar nódulos pulmonares no contexto $2 \mathrm{D}$ que seja tão eficaz quanto aqueles propostos na literatura, mas com menor 
custo computacional.

\section{Materiais e Métodos}

Nesta seção, apresentamos o método proposto para detecção de nódulos pulmonares em imagens de TC. Nosso método é dividido em duas etapas principais: (i) segmentação da região do parênquima pulmonar, bem como a divisão da região segmentada em fragmentos da imagem (patches) e (ii) identificação de nódulos dentro do parênquima a partir da classificação dos patches em nódulo ou não-nódulo. A etapa de extração do parênquima é realizada com uma rede U-Net. A etapa de detecção do nódulo é realizada por meio do uso de uma CNN agregada à redução de dimensionalidade de dados utilizando-se o algoritmo PCA. O uso da técnica de redução de dimensionalidade se deu com o objetivo de otimizar a eficácia do método por meio do filtro de características menos relevantes pela CNN. A Figura 1 ilustra as etapas do método proposto.

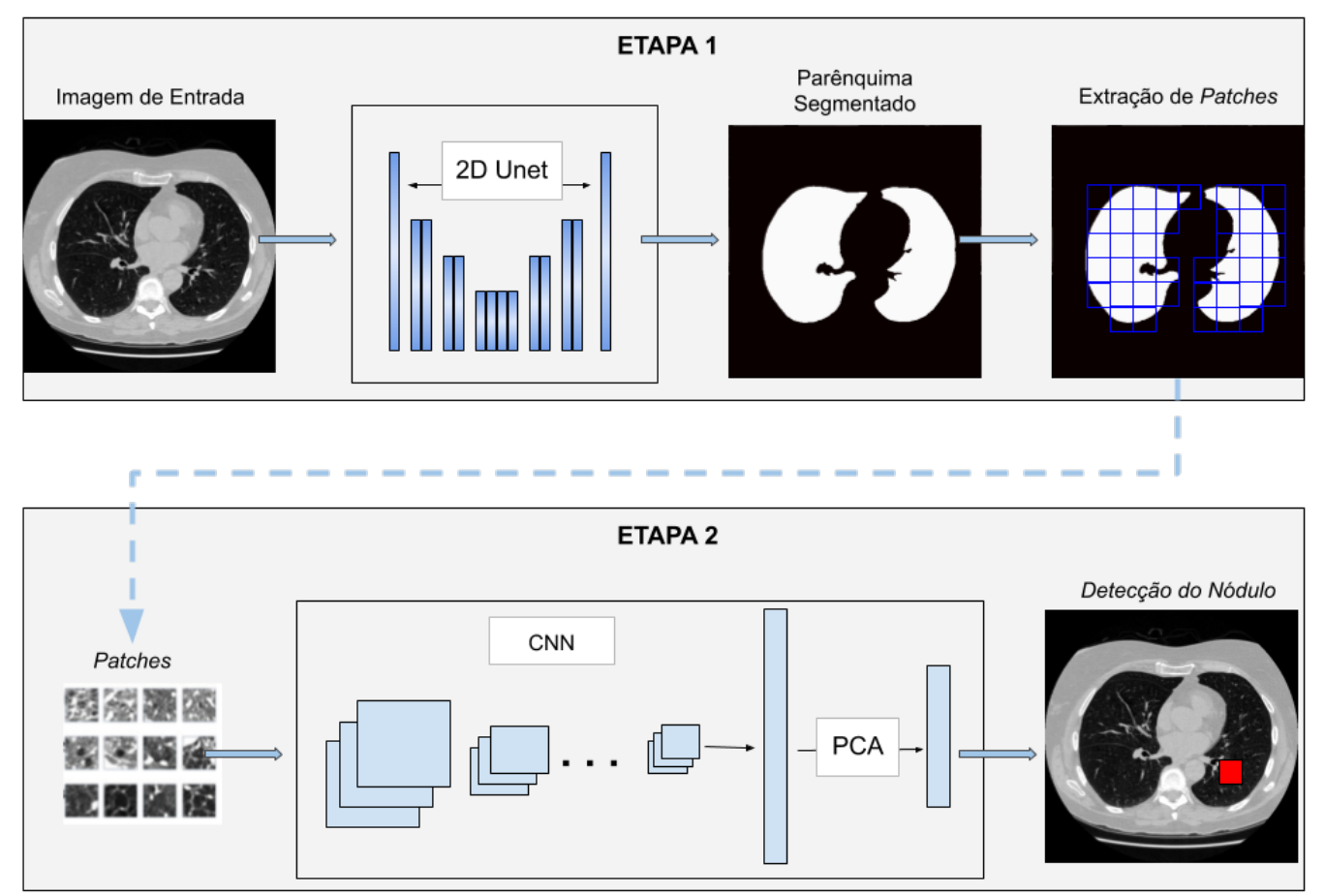

Figura 1. Visão geral do método proposto.

\subsection{Segmentação da Região do Parênquima}

O parênquima pulmonar é um tecido que envolve o pulmão, e consiste na compressão de um grande número de alvéolos, formando uma enorme superfície com a função de manter a troca de gases [Suki et al. 2011]. A incidência de nódulos pulmonares ocorre sempre próxima ao parênquima [Suki et al. 2011]. Considerando este fato, a primeira etapa do método proposto é a remoção de áreas da imagem que são externas ao parênquima. $O$ objetivo dessa remoção é diminuir o espaço de busca por possíveis nódulos, de modo a reduzir o custo computacional e diminuir a possibilidade de falsos positivos. 
A U-Net é uma rede neural convolucional que foi originalmente criada para segmentação de estruturas em imagens biomédicas. Ela se assemelha a uma arquitetura típica de uma CNN. Contudo, não apresenta uma sequência de camadas totalmente conectadas, como geralmente se é usado. A rede consiste em uma etapa de contração seguida de uma etapa de expansão, o que fornece a arquitetura em forma de $U$. Na etapa de contração ocorre uma diminuição da dimensão dos dados como também a extração de características importantes para a criação da máscara da estrutura fisiológica. Já na etapa de expansão é usado das características extraídas para a criação de uma nova imagem, onde o valor de cada pixel é a probabilidade dele pertencer à estrutura a ser segmentada. A descrição detalhada da rede U-Net pode ser encontrada em [Ronneberger et al. 2015].

Para implementar a primeira etapa do método proposto foi preciso ser feita a rotulação da região do parênquima de várias imagens para treinar a rede U-Net. Este processo foi realizado forma manual sobre uma amostra de 1000 imagens, onde cada imagem de TC teve o parênquima segmentado afim de serem criadas máscaras para o treinamento da U-net e desta forma ser possível a obtenção de uma rede capaz de segmentar o parênquima pulmonar. Tal processo de rotulação foi realizada com o auxílio de uma ferramenta open source [Bréhéret 2017] que implementa o algoritmo watershed [Zhang et al. 2010].

Na sequência utilizamos o modelo da U-Net na classificação pixel-a-pixel para resolver um problema de duas classes: pertence ou não à região dentro do parênquima. Nesse sentido, os rótulos associados às imagens são representados por máscaras binárias (ground truth) de iguais dimensões à imagem original. Os valores de intensidade dos pixels (0 ou 1) presentes na máscaras indicam a que classe cada pixel pertence. A rede U-net é então treinada para realizar essa segmentação de forma automática nas imagens originais. Alguns resultados relativos à segmentação da região do parênquima utilizando a U-net podem ser vistos na Figura 2. Descrições sobre a base de imagens utilizada, bem como resultados quantitativos que apontam a acurácia deste modelo serão discutidos na Seção 4.4.
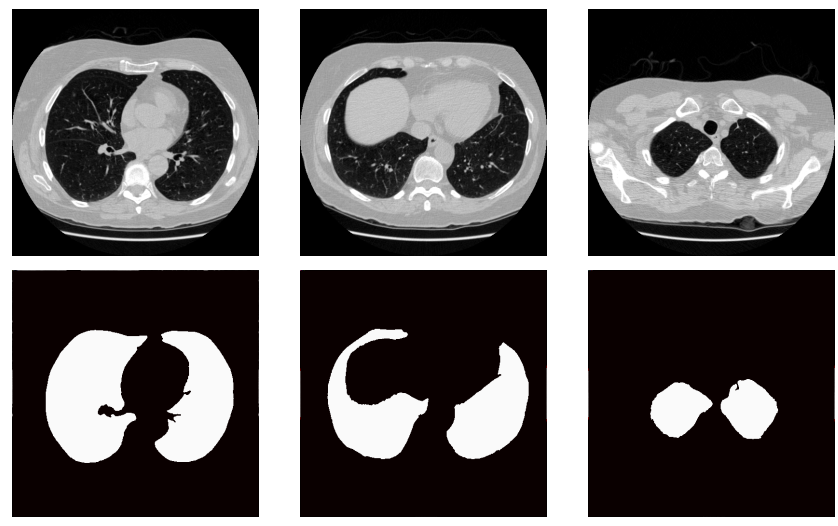

Figura 2. Segmentação da região do parênquima. Na linha superior são exibidas as imagens originas. Na linha inferior são exibidas as respectivas máscaras de segmentação produzidas pela U-net. 


\subsection{Detecção de Nódulos com CNN + PCA}

A segunda etapa do método proposto consiste na identificação dos nódulos existentes na região segmentada. Foi decidido pelo uso de CNNs 2D em vez de 3Ds de modo a priorizar custo computacional reduzido e menor complexidade do modelo sem a perda de precisão ([Xie et al. 2019, Eun et al. 2018]). Assim, uma CNN 2D é treinada para classificar os patches em contendo ou não nódulo.

Devido às dimensões dos nódulos no dataset utilizado (maiores ou iguais a $3 \mathrm{~mm}$ ), utilizamos como abordagem a divisão em grid da região segmentada do parênquima pulmonar. Dado que o tamanho das imagens originais serem no formato 512x512, para que a divisão seja possível foi estudado as possíveis granularidades para os patches: $64 \times 64$, 32x32 e 16x16. O tamanho 64x64 permitiu melhores resultados em testes preliminares. Acreditamos que isso ocorre devido ao fato deste formato possuir menor perda do contexto do nódulo. Tais patches são usados como entrada para a CNN que irá predizer a probabilidade de existência de nódulo sólido em um dado patch. Para fins de balanceamento de classes no dataset de treino, foi selecionada a mesma quantidade de patches que possuem e não possuem nódulo. A arquitetura e os hiper-parâmetros da CNN utilizada foram definidos de forma empírica com base na performance de treino e validação. A arquitetura é mostrada na Figura 3.

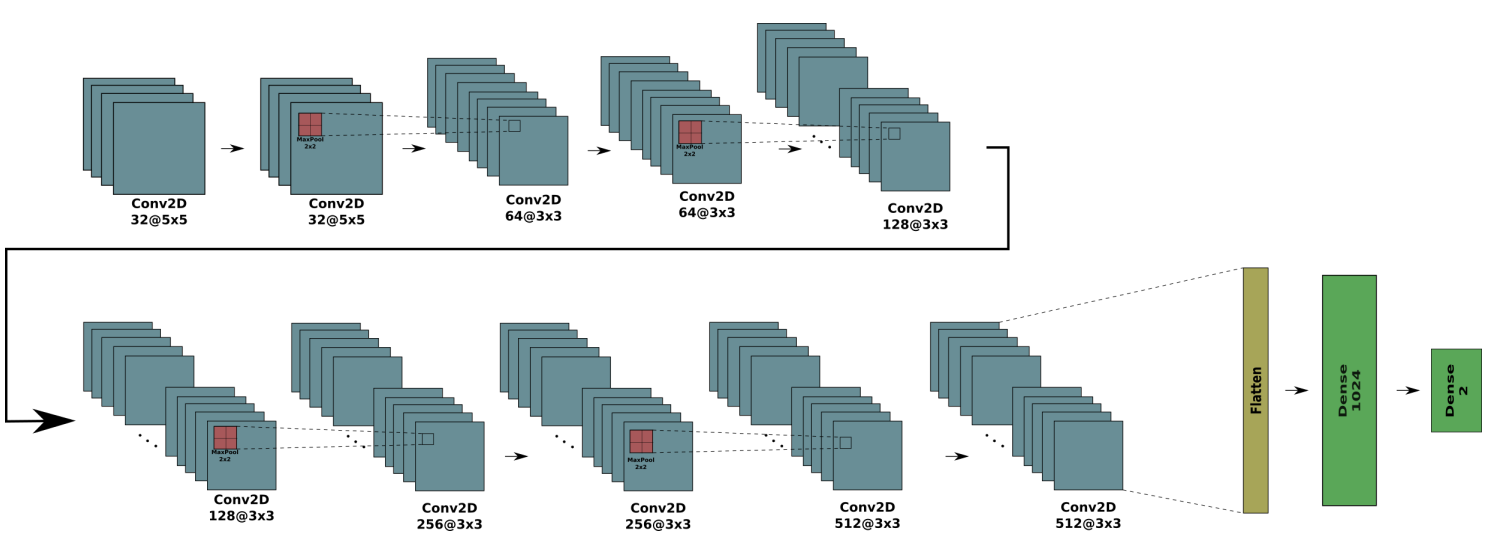

Figura 3. Arquitetura da CNN utilizada no processo de detecçâo de nódulos

Foi utilizado a função de ativação ReLU nas camadas de convolução e na camada densa. Não foi usado padding nas camadas de convolução. Nas camadas de MaxPooling foi usado stride de $2 \times 2$. Entre as camadas densas foi usado Dropout com a probabilidade de $25 \%$ de permanência dos neurônios, e na última camada densa foi usado softmax para previsão da probabilidade de existência de nódulo.

Como na escala dos patchs (64x64 pixels) não se tem uma diferença notável entre algumas estruturas fisiológicas e regiões nodulares, a CNN extrai muitas características redundantes para a identificação do nódulo. Logo, pelo mesmo motivo que [Chen et al. 2014], onde o classificador final recebe uma entrada com grandes dimensões contendo um alto índice de redundância, optamos por aplicar o PCA antes da última camada. Onde nós utilizamos da técnica de transfer learning, caraterizado pela reutilização do modelo aprendido pela CNN, sem necessidade de treina-la novamente.

O uso do PCA se dá pelo mesmo motivo de [Chen et al. 2014], onde o classificador final recebe uma entrada com grandes dimensões, contendo um grande índice de 
redundância. As características extraídas pela CNN, são transformadas pelo PCA, que reduz a dimensão dos dados em 50\%. Em seguida essas serão a entrada de uma rede totalmente conectada (Multilayer Perceptron - MLP), que possui a mesma arquitetura da parte denotada como Dense da Figura 3, para previsão da probabilidade de existência de nódulo no patch analisado.

\section{Experimentos e Resultados}

\subsection{Base de Dados}

Para a condução dos experimentos, foi utilizado como base de dados o LUNA16 [Setio et al. 2017], que é um subset do LIDC (Lung Image Database Consortium) [Armato III et al. 2011]. A base de dados do LUNA16 possui um total de 888 exames, sendo que estes exames possuem diferentes números de imagens transversais (slices) do pulmão. Foram mantidos na base apenas exames com nódulos maiores que $3 \mathrm{~mm}$ e que foram classificados como nódulo por pelo menos 3 dos 4 radiologistas. Dentre esses exames, existem cerca de 1557 nódulos catalogados com suas respectivas coordenadas centrais.

\subsection{Métricas}

Foram utilizadas como métricas de avaliação da qualidade do método proposto a acurácia (Equação 1) e a sensibilidade (Equação 2) na classificação dos patches. A sensibilidade é calculada como a porcentagem de casos com nódulo que foram diagnosticados corretamente pelo método, sendo calculada como a razão do número de verdadeiros positivos preditos pela soma de verdadeiros positivos com falsos negativos (FN). A acurácia descreve a precisão do método tanto para casos positivos quanto para casos negativos e corresponde à razão da soma do número de verdadeiros positivos (TP) com o de verdadeiros negativos (TN) pelo total de previsões. Tais métricas foram empregadas em vários artigos na literatura (ver Tabela 1).

$$
\begin{gathered}
\text { Acurácia }=\frac{T P+T N}{T+P} \\
\text { Sensibilidade }=\frac{T P}{T P+F N}
\end{gathered}
$$

\subsection{Treinamento e Validação}

Com um total de 1327 scans, foi selecionado, de maneira aleatória 266 scans para validação e 1061 scans para o treino da U-net para a segmentação do parênquima. Como forma de validação utilizamos o processo de validação cruzada com 10 fold. A acurácia média da validação foi de $95,97 \%$ na sobreposição dos pixels no processo de segmentação da região do parênquima.

No processo de validação do método de identificação dos nódulos foram realizados os processos descritos nas Seções 3.1 e 3.2. Usamos patchs com dimensões de 64 x 64 pixels. O processo de valiação cruzada 10-fold foi aplicado a 3388 patches, onde metade deste conjunto possui nódulos e outra metade não.

Os valores dos hiper-parâmetros do método foram selecionados empiricamente utilizando a acurácia como critério de escolha. Para a taxa de aprendizado foram realizados testes preliminares com os valores $1 \times 10 \mathrm{e}-4,1 \times 10 \mathrm{e}-5$ e $1 \times 1 \mathrm{e}-6$. Para o batch size 
foram testados os valores 10,15 e 20 . Já para o número de épocas os valores testados foram: 100,200 e 300.

Para o método utilizado, foi escolhido o algoritmo de otimização Adam para o treinamento, a taxa de aprendizado utilizada foi $1 \times 10 \mathrm{e}-5$, a entropia-cruzada binária ( $\mathrm{bi}$ nary crossentropy) foi utilizada como função de erro, o batch size foi definido com valor 20 e o número de épocas definido com valor 300 .

\subsection{Resultados}

Em um primeiro experimento, comparamos a eficácia na detecção de nódulos produzida pela CNN simples e a CNN+PCA proposta nesse trabalho. Ambas as redes possuem exatamente a mesma arquitetura. Como apresentado na Tabela 2, nas duas primeiras linhas, mostramos a comparação dos resultados obtidos com aplicação do método proposto. Estes resultados mostram uma melhora significativa na eficácia da identificação, tanto em termos de sensibilidade quanto em termos de acurácia. Essa melhoria ocorre pois o PCA é capaz de eliminar características redundantes ou com baixa relevância nas imagens antes da camada completamente conectada da CNN. As características 50\% mais importantes identificadas pelo PCA são selecionadas na classificação final.

A Tabela 2 mostra um comparativo dos resultados obtidos pelo método proposto com trabalhos relacionados que utilizaram como base de dados o LUNA16 (subset do LIDC) ou o LIDC, que foram aplicados à imagens 2D. Vale ressaltar que todos os métodos aqui analisados e comparados fizeram uso do mesmo métodos de validação, o $K$-fold. Analisando os resultados, percebe-se que nossa proposta superou [Eun et al. 2018] em acurácia e [Jiang et al. 2018] em termos de sensibilidade (não usou a acurácia), tendo em vista que o último usa uma estrutura mais complexa que a proposta neste trabalho. $\mathrm{O}$ trabalho proposto em [Xie et al. 2019] apresentou os melhores resultados. Mesmo não superando [Xie et al. 2019] em ambas as métricas, o nosso trabalho possui uma estrutura simples em relação aos trabalhos comparados. Devido à essa simplicidade estrutural, o modelo proposto é mais eficiente para a identificação em contexto prático.

Tabela 2. Tabela comparativa de resultados

\begin{tabular}{|l|l|c|c|}
\hline Trabalho & Método & Acurácia & Sensibilidade \\
\hline CNN (Proposto) & U-net + CNN 2D & $76,36 \%$ & $74,40 \%$ \\
\hline CNN + PCA (Proposto) & U-net + CNN 2D + PCA & $84,00 \%$ & $86,08 \%$ \\
\hline [Eun et al. 2018] & Autoencoder + K-means + CNN 2D & $80,00 \%$ & $92,20 \%$ \\
\hline [Jiang et al. 2018] & CNN 2D Multi-escala & - & $80,06 \%$ \\
\hline [Xie et al. 2019] & Faster R-CNN + CNN 2D & $86,42 \%$ & $74,40 \%$ \\
\hline
\end{tabular}

\section{Conclusão}

O câncer de pulmão merece uma atenção especial dos pesquisadores devido a sua alta incidência e alta taxa de mortalidade. Diante disto, neste trabalho, investigamos como detectar de forma automática a presença de nódulos pulmonares em imagens de tomografia computadorizada do tórax do paciente.

A abordagem proposta utiliza duas tecnologias de aprendizado profundo apresentadas na literatura, a saber as redes U-Net e CNN. A Análise de Componentes Principais 
foi inserida na estrutura da CNN de modo a refinar os resultados de classificação da rede, diminuindo o custo computacional e o tempo de obtenção para um diagnóstico. Os resultados demonstraram que o método proposto alcançou $86,08 \%$ de sensibilidade, o que demonstra que a abordagem é competitiva em relação aos trabalhos que são considerados o estado-da-arte, que em sua maioria trabalham em um nível de complexidade 3D.

A presente pesquisa pode ser expandida de várias formas. Um trabalho futuro pode investigar o aumento da base de dados com uso de outras técnicas de Aprendizado Profundo como GANs, de modo a melhorar o processo de treinamento e, em consequência, a acurácia do método. Uma outra possibilidade de pesquisa futura é o uso da abordagem multi-escala, onde patches com diferentes tamanhos podem ser usados como entrada para a rede. O objetivo dessa abordagem é corrigir o problema de haver nódulos com diferentes tamanhos nos exames e estes podem não ser corretamente identificados em patches de tamanho fixo.

\section{Referências}

Armato III, S. G., McLennan, G., Bidaut, L., McNitt-Gray, M. F., Meyer, C. R., Reeves, A. P., Zhao, B., Aberle, D. R., Henschke, C. I., Hoffman, E. A., et al. (2011). The lung image database consortium (lidc) and image database resource initiative (idri): a completed reference database of lung nodules on ct scans. Medical physics, 38(2):915931.

Bogot, N. R., Kazerooni, E. A., Kelly, A. M., Quint, L. E., Desjardins, B., and Nan, B. (2005). Interobserver and intraobserver variability in the assessment of pulmonary nodule size on ct using film and computer display methods1. Academic Radiology, 12(8):948-956.

Bréhéret, A. (2017). Pixel Annotation Tool. https: / / github.com/abreheret/ PixelAnnotationTool.

Cesár Uehara, Sérgio Jamnik, I. L. S. (1998). Cancêr de Paulmão.

Cesár Uehara, Sérgio Jamnik, I. L. S. E. L. B. W. V. d. S. (2008). Características clínicas, diagnosticas e laboratoriais de portadores de carcinoma bronquioloaveolar.

Chen, G., Zhang, J., Zhuo, D., Pan, Y., and Pang, C. (2019). Identification of pulmonary nodules via ct images with hierarchical fully convolutional networks. Medical \& Biological Engineering \& Computing, 57(7):1567-1580.

Chen, Y., Lin, Z., Zhao, X., Wang, G., and Gu, Y. (2014). Deep learning-based classification of hyperspectral data. IEEE Journal of Selected Topics in Applied Earth Observations and Remote Sensing, 7(6):2094-2107.

Ding, Jia Li, A. H. Z. W. L. (2019). Accurate pulmonary nodule detection in computed tomography images using deep convolutional neural networks. Lecture Notes in Computer Science (including subseries Lecture Notes in Artificial Intelligence and Lecture Notes in Bioinformatics), 10435 LNCS:559-567.

Dunteman, G. H. (1989). Principal components analysis. Number 69. Sage.

Eun, H., Kim, D., Jung, C., and Kim, C. (2018). Single-view 2d cnns with fully automatic non-nodule categorization for false positive reduction in pulmonary nodule detection. Computer Methods and Programs in Biomedicine, 165:215 - 224. 
Golan, R., Jacob, C., and Denzinger, J. (2016). Lung nodule detection in ct images using deep convolutional neural networks. In 2016 International Joint Conference on Neural Networks (IJCNN), pages 243-250.

Gu, Y., Lu, X., Yang, L., Zhang, B., Yu, D., Zhao, Y., Gao, L., Wu, L., and Zhou, T. (2018). Automatic lung nodule detection using a 3d deep convolutional neural network combined with a multi-scale prediction strategy in chest cts. Computers in Biology and Medicine, 103:220 - 231.

Huang, Xiaojie Shan, J. V. V. (2017). Lung nodule detection in ct using 3d convolutional neural networks. pages 379-383.

Jiang, H., Ma, H., Qian, W., Gao, M., and Li, Y. (2018). An automatic detection system of lung nodule based on multigroup patch-based deep learning network. IEEE Journal of Biomedical and Health Informatics, 22(4):1227-1237.

Ker, J., Wang, L., Rao, J., and Lim, T. (2017). Deep learning applications in medical image analysis. Ieee Access, 6:9375-9389.

Lakshmanaprabu S.K., Sachi Nandan Mohanty, S. K. A. N. G. R. (2018). Optimal deep learning model for classification of lung cancer on ct images. Future Generation Computer Systems (2018).

Rakhlin, A., Shvets, A., Iglovikov, V., and Kalinin, A. A. (2018). Deep convolutional neural networks for breast cancer histology image analysis. In International Conference Image Analysis and Recognition, pages 737-744. Springer.

Ren, S., He, K., Girshick, R., and Sun, J. (2015). Faster r-cnn: Towards real-time object detection with region proposal networks.

Ronneberger, O., Fischer, P., and Brox, T. (2015). U-net: Convolutional networks for biomedical image segmentation. CoRR, abs/1505.04597.

Setio, A. A. A., Traverso, A., [de Bel], T., Berens, M. S., van den Bogaard, C., Cerello, P., Chen, H., Dou, Q., Fantacci, M. E., Geurts, B., van der Gugten, R., Heng, P. A., Jansen, B., [de Kaste], M. M., Kotov, V., Lin, J. Y.-H., Manders, J. T., Sóñora-Mengana, A., García-Naranjo, J. C., Papavasileiou, E., Prokop, M., Saletta, M., Schaefer-Prokop, C. M., Scholten, E. T., Scholten, L., Snoeren, M. M., Torres, E. L., Vandemeulebroucke, J., Walasek, N., Zuidhof, G. C., van Ginneken, B., and Jacobs, C. (2017). Validation, comparison, and combination of algorithms for automatic detection of pulmonary nodules in computed tomography images: The luna16 challenge. Medical Image Analysis, 42:1 - 13.

Shen, D., Wu, G., and Suk, H.-I. (2017). Deep learning in medical image analysis. Annual review of biomedical engineering, 19:221-248.

Suki, B., Stamenović, D., and Hubmayr, R. (2011). Lung Parenchymal Mechanics, pages 1317-1351. American Cancer Society.

Winkels, M. and Cohen, T. S. (2019). Pulmonary nodule detection in ct scans with equivariant cnns. Medical Image Analysis, 55:15 - 26.

Xie, H., Yang, D., Sun, N., Chen, Z., and Zhang, Y. (2019). Automated pulmonary nodule detection in ct images using deep convolutional neural networks. Pattern Recognition, $85: 109-119$. 
Zhang, M., Zhang, L., and Cheng, H. (2010). A neutrosophic approach to image segmentation based on watershed method. Signal Processing, 90(5):1510 - 1517. Special Section on Statistical Signal Array Processing. 